\title{
First Study Treatment Time
}

National Cancer Institute

\section{Source}

National Cancer Institute. First Study Treatment Time. NCI Thesaurus. Code C162169.

The time of the subject's first study treatment. 